\title{
Grain Boundary Responses to Heterogeneous Deformation in Tantalum Polycrystals
}

\author{
THOMAS R. BIELER, ${ }^{1,3}$ SCOTT C. SUTTON,${ }^{1}$ BRET E. DUNLAP,${ }^{1}$ \\ ZACKERY A. KEITH, ${ }^{1}$ PHILIP EISENLOHR, ${ }^{1}$ MARTIN A. CRIMP, ${ }^{1}$ \\ and BRAD L. BOYCE ${ }^{2}$ \\ 1.-Chemical Engineering and Materials Science, Michigan State University, East Lansing, \\ MI 48824, USA. 2.—Sandia National Laboratory, Albuquerque, NM 87185, USA. 3.—e-mail: bieler@ \\ egr.msu.edu
}

\begin{abstract}
The evolution of heterogeneous deformation in a tantalum polycrystal was examined during a three-point bending experiment using electron backscatter pattern mapping. Slip bands formed at strains as low as $1 \%$, and they became more intense with strain. Heterogeneous deformation was evident as intragranular orientation gradients as large as $30^{\circ}$ were observed after a strain of about $8 \%$. Nonmonotonic changes in the local average misorientation distribution were observed, implying that dislocation substructure developed in a complex manner. Slip bands were analyzed using plane traces computed from local orientation information. With the assumption of uniaxial stress, Schmid factors for favorable slip systems were identified for each grain and compared with observations, showing evidence for macroscopic activity on both $\{110\}$ and $\{112\}$ slip systems. Reconstructed boundary data were used to estimate the geometric potential for slip transfer at grain boundaries. The correlations indicated that when active slip systems were favorably oriented for slip transfer across the boundary, it was often observed in the form of continuous slip bands aligned across the boundary. In boundaries where geometrical alignment and Schmid factors were not favorable for slip transfer, there was a higher likelihood to form ledges (topographic discontinuities) along the grain boundaries. Dislocation pileups at grain boundaries were also correlated with a low potential for slip transfer.
\end{abstract}

\section{INTRODUCTION}

Damage nucleation is an important frontier in materials science, as understanding this process quantitatively and mechanistically is necessary for predicting ductility and lifetimes. In many practical alloys, hard second-phase particles are preexisting damage nucleation sites, but in single-phase metals or multiphase alloys containing only ductile phases, damage often nucleates at grain or phase boundaries. Prior studies of damage nucleation at grain boundaries show that the ease of slip transfer and the magnitude of residual defect content left in grain boundaries influences boundary energy and, hence, cohesion and damage nucleation. ${ }^{1-6}$ Thus, the interactions of dislocations with grain boundaries must be quantitatively understood and modeled accurately during heterogeneous deformation before damage nucleation mechanisms can be introduced into models capable of predicting the onset of fracture.

It follows that to develop a fundamental basis to understand the damage nucleation, there is a need to quantitatively assess grain boundary resistance to dislocation transmission in deforming materials. One approach to understand the nature of slip transfer across grain boundaries is to use the geometric relationships between slip systems on either side of a grain boundary with the parameter $m^{\prime}=\cos \psi \cos \kappa$, with $\psi$ the angle between a pair of slip plane normals and $\kappa$ the angle between a pair of slip directions. ${ }^{7,8}$ As this parameter only considers the compatibility of different slip systems across a boundary, but not the activation of those slip systems, it needs to be combined with an assessment of the activity of slip systems. The most natural way to 
do this is by using the Schmid factor $m$, i.e., with knowledge of activated slip systems, in combination with $m^{\prime},{ }^{9,10}$

The identification of active slip systems in bodycentered cubic (bcc) metals is complicated due to the dislocation core relaxation of screw dislocations onto multiple potential slip planes. ${ }^{11}$ First-principles atomic simulations of slip in Ta predict no $\{112\}$ slip at $0 \mathrm{~K}^{11}$ However, slip on both $\{110\}$ and $\{112\}$ planes has been identified in many bcc metals. A detailed study of slip phenomena in $\mathrm{Nb}$ single crystals implies slip on $\{112\}$ planes above $150 \mathrm{~K} .{ }^{12}$ Texture studies also imply slip on $\{112\}$ planes in $\mathrm{Nb}$ and $\mathrm{Ta}^{13}$ Recent studies on large oligo crystal samples deformed at room temperature show stronger evidence for $\{110\}$ slip than $\{112\}$ slip. ${ }^{14}$ Thus, there is not yet a clear consensus about the role of $\{112\}$ slip in Ta.

The processes that lead to damage in Ta will depend on details of how slip interacts with grain boundaries. To identify mechanisms responsible for heterogeneous deformation that arise due to differential deformation in differently oriented grains, bending experiments were conducted on a Ta polycrystal. This deformation mode puts the maximum stress on a surface that can be readily characterized using scanning electron microscopes and associated orientation analysis, in contrast to tensile tests, where fatal flaws usually develop unseen inside the neck region. ${ }^{15}$

\section{EXPERIMENTAL PROCEDURES}

A sheet of $99.9 \%$ pure polycrystalline Ta, provided by Sandia National Laboratory, was used to investigate microstructure evolution during sequential deformation in a bending stage. The samples described here were $30 \times 3 \times 1.3 \mathrm{~mm}^{3}$ and cut from the sheet so that the long (tensile) direction was aligned with the original sheet transverse direction. One $30 \times 3 \mathrm{~mm}^{2}$ side was mechanically polished using decreasing grit sizes, with final polishing using colloidal silica.

The initial state of the microstructure was characterized using scanning electron microscopy. Backscattered and secondary electron images were taken at several different magnifications to identify the initial grain size and surface features. These images were used to establish the zero strain reference state by measuring distances between a prescribed set of triple points at least $500 \mu \mathrm{m}$ apart. Electron backscattered diffraction pattern (EBSP) mapping was done using EDAX/TSL Orientation Imaging Microscopy (EDAX, Inc., Mahwah, NJ) systems on a JEOL 6500F (JEOL Ltd., Tokyo, Japan) and a Tescan Mira3 (TESCAN ORSAY HOLDING, a.s., Brno-Kohoutovice, Czech Republic). A region measuring approximately $2.5 \times$ $1 \mathrm{~mm}^{2}$ was mapped prior to deformation. This map indicated that the vast majority of the grains were recrystallized, with only a few grains showing orientation gradients, which is indicative of recovery. The polish was sufficiently good to obtain an average confidence index of 0.85 and no unindexed pixels.

The specimen was deformed using a manually loaded three-point bending fixture shown in Fig. 1. The center pin had a radius of curvature of $1.5 \mathrm{~mm}$, which limited the maximum strain at the surface to about $10 \%$ tensile strain. SEM measurements were retaken at several increments in strain on the loaded specimen. Orientation mapping scans were made on the same area using the same step size used in the initial measurement. While photographs of the bent specimen in the stage were taken to measure the radius of curvature with sequential increments in strain to approximate the strain, local surface strains were measured by tracking relative displacements of multiple triple junctions identified in the undeformed state, and averaged. These latter strain measurements are reported in this study.

With increasing strain, the number of nonindexed or low-confidence indexation pixels increased slightly, with no more than $0.2 \%$ nonindexed pixels and no more than $2 \%$ of the pixels with a confidence index less than 0.05 . Consequently some cleanup was done to reduce the number of low confidence or unindexed pixels, first using a confidence index based cleanup, and second, a dilation to remove 'grains' that contained less than 30 pixels (smaller than $4 \mu \mathrm{m}$ diameter), in order to reduce excessive numbers of boundaries in reconstructed boundary files. This set of measurements was repeated on a different specimen to confirm repeatability of measured quantities, as discussed below.

\section{RESULTS}

Electron backscattered pattern diffraction (EBSD) normal direction inverse pole figure maps of the initial and final microstructure in the same location are shown in Fig. 2. There is a fairly wide grain size distribution from $10 \mu \mathrm{m}$ to $100 \mu \mathrm{m}$. There are clearly two dominant colors present, red and blue, which are related to the texture of the sample. The EBSD-based pole figures shown in Fig. 3 are based on the maps and include information from approximately 1000 grains. The initial texture

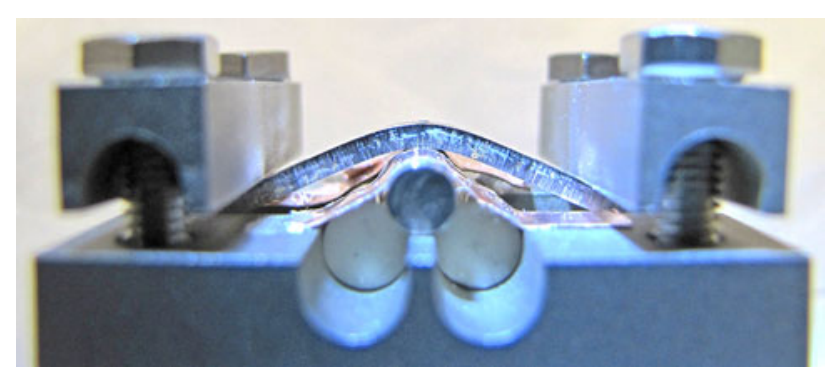

Fig. 1. Three-point bending of a Ta polycrystal using a four-point bend apparatus with an extra rod to achieve sufficiently large strain in the middle of the sample. 

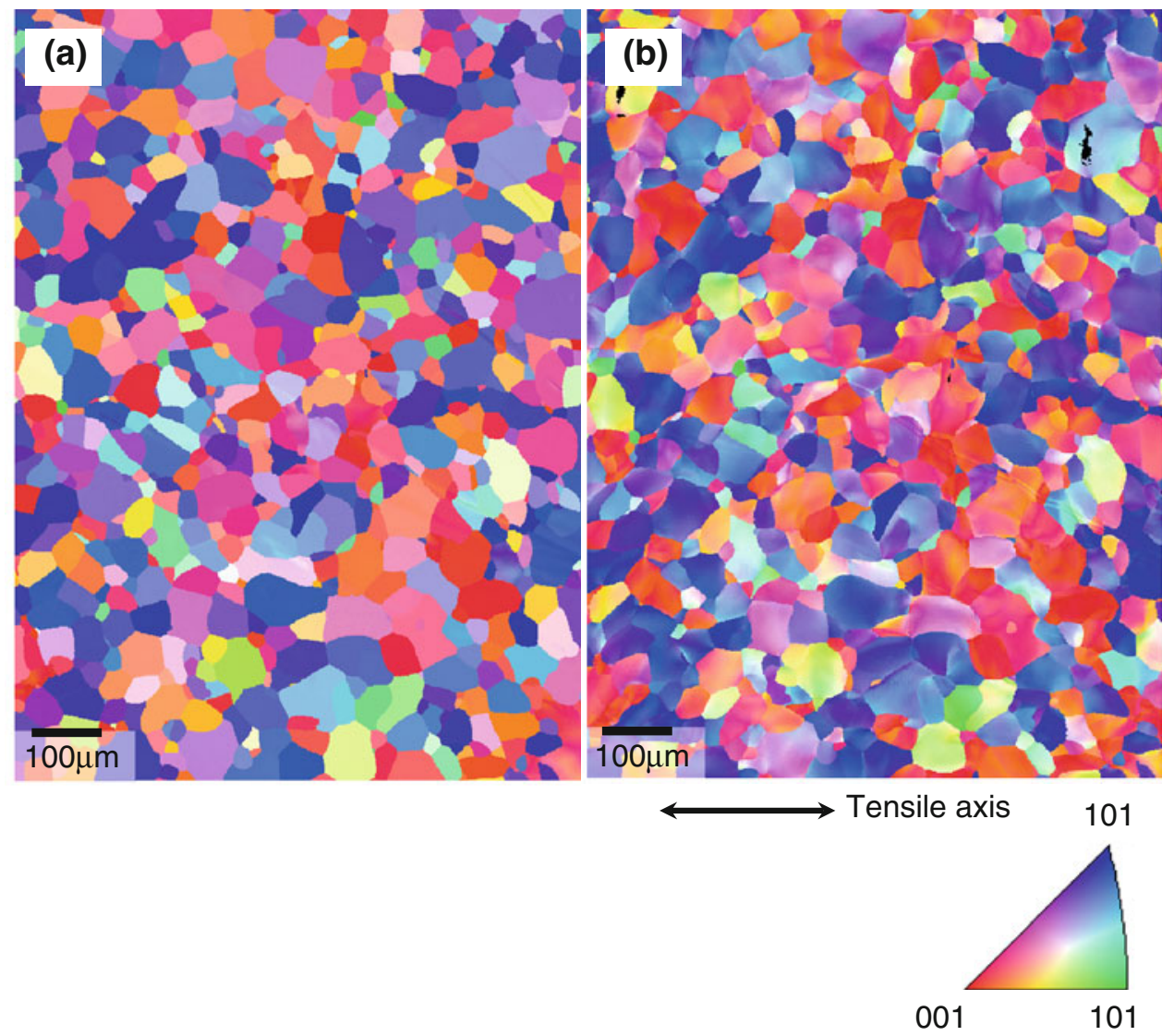

Fig. 2. (a) Sheet normal direction inverse pole figure maps showing the red rotated cube and blue 111 fiber texture components in the undeformed sample (a), and (b) orientation gradient development after $7.9 \%$ strain.

shows a typical bcc rolling texture, with [110](111) and [110](001) components with $\sim 4$ times random intensities. With straining in the transverse (horizontal) direction, the fraction of the $\{111\}$ fiber component increased slightly, making a more even ring of density around the center that is most evident in the $\{111\}$ pole figure.

The grain reference orientation deviation map in Fig. 4a illustrates the homogeneity of the initial orientations within each grain. Most of the grains are uniformly white, indicating that the entire grain is within a degree of the average orientation. Some grains have low-angle boundaries with neighboring grains, leading to a uniform shade of gray in one or more parts of the grain(s). Also, a few grains show orientation gradients (e.g., lower right corner), which are representative of grains that did not recrystallize. With increasing strain, many grains develop orientation (color) gradients (Figs. 2b, 4b). The grain reference orientation map in Fig. 4b shows very few grains with a uniform shade of gray. The range of orientation spread present in a typical grain is about $10^{\circ}$, but some grains show gradients up to about $30^{\circ}$. As made evident in the magnified insets, the orientation spread varies monotonically with distance toward grain boundaries, indicating that the geometrically necessary dislocation density increases monotonically.
The evolution of intergranular disorientation and intragranular misorientation* with strain in the region examined is illustrated in Fig. 5a and b, respectively. Figure 5a shows the probability distribution of intergranular disorientation between orientation pixels (spaced $700 \mathrm{~nm}$ ) for angles greater than $2.5^{\circ}$ (avoiding contributions from intragranular misorientations). The major change with strain in the distribution is the increase at disorientation values below $5^{\circ}$ (Fig. 5a), indicating the development of subboundaries. Figure 5b displays the EBSD measured local average misorientation (LAM; based on pixels up to the second neighbor), which is a measure of the local lattice curvature, and hence is indicative of the presence of geometrically necessary dislocations. A perfect crystal is expected to have a high and narrow peak as far to the left as the precision of the EBSD measurement allows, generally accepted to be $1^{\circ}$ or smaller. In the undeformed case, the peak is at

*Disorientation is used to describe the angle of mismatch between two grains, at the grain boundary. The word misorientation is used to refer to the difference in orientation between two locations within a grain, and hence, it is descriptive of lattice curvature and low-angle boundaries. The two ideas overlap with low-angle boundaries. 

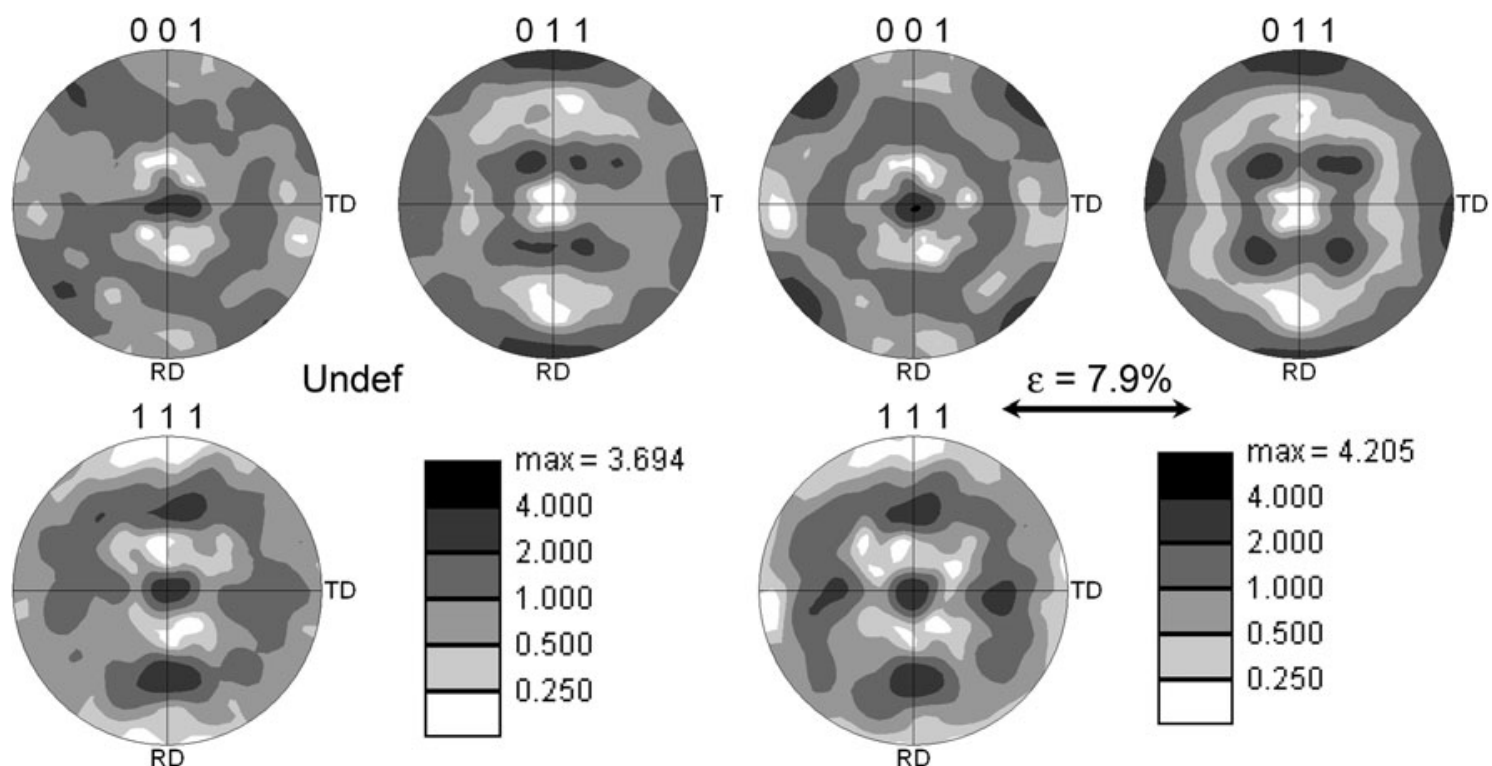

$\max =3.694$

4.000

2.000

1.000

0.500

0.250

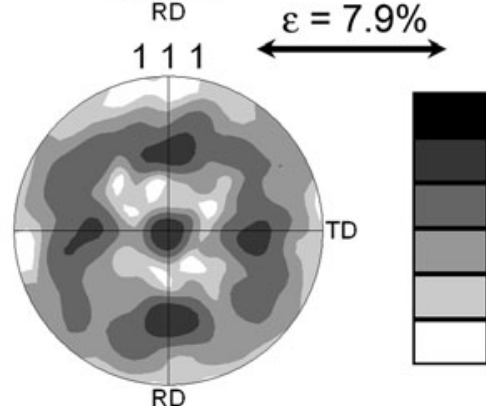

$\max =4.205$

4.000

2.000

1.000

0.500

0.250

Fig. 3. Texture from EBSD maps illustrating the change in texture with deformation; the two components of texture intensified with strain in the horizontal transverse direction, a rotated cube and a $\langle 111\rangle$ fiber about the original surface normal direction.

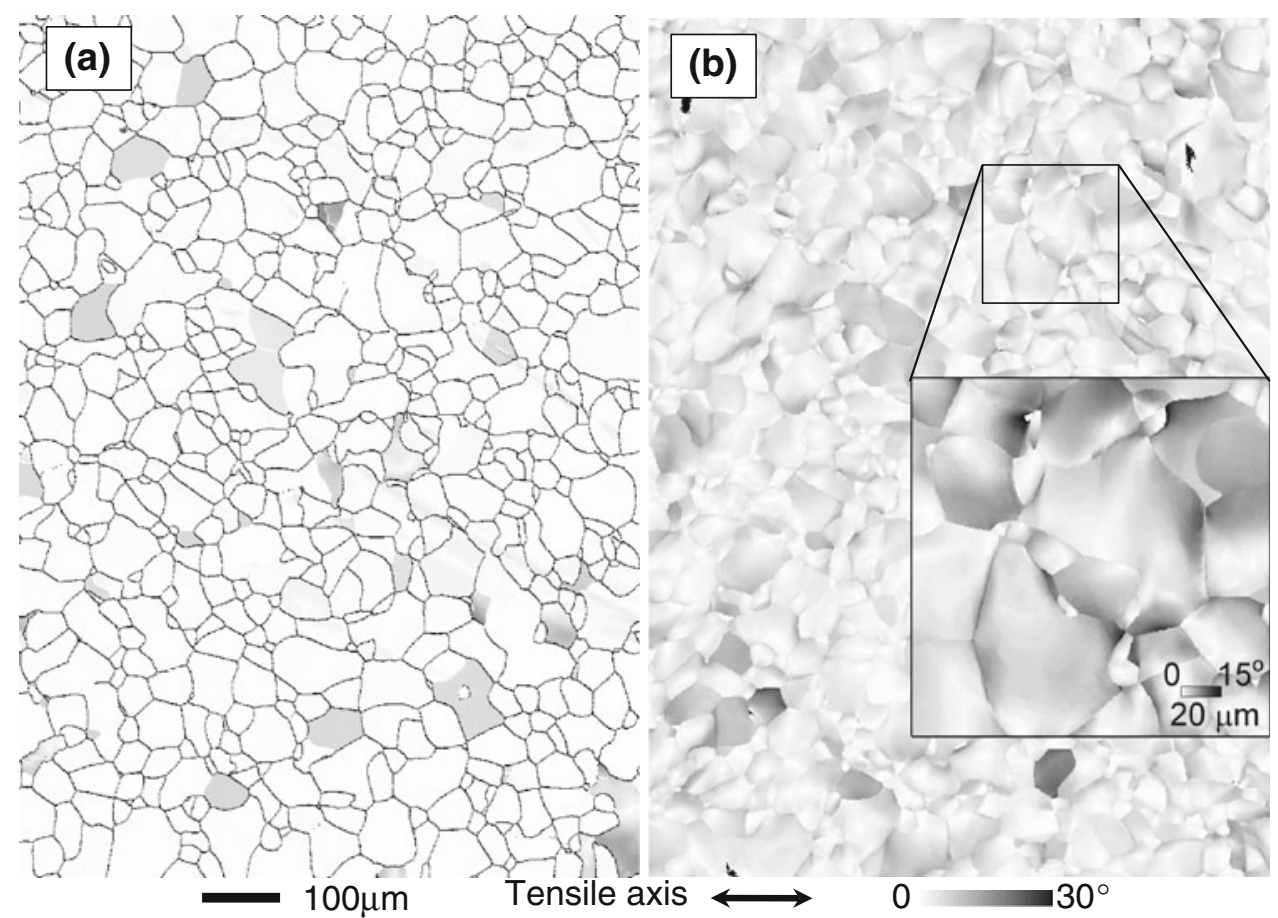

Fig. 4. Grain reference orientation deviation maps based on the minimum kernel average misorientation in each grain show that most grains do not have orientation gradients in undeformed (a), while orientation gradients are typical in the deformed map at a strain of $7.9 \%$ (b); inset shows enlargement showing monotonic increase in deviation toward the grain boundary.

$0.25^{\circ}$, which may reflect improved accuracy due to averaging effects. With strain increasing up to $2 \%$, the distribution shifts and becomes broader. With further strain, the distribution narrows again. This effect was confirmed using a second sample, as shown in the inset in Fig. 5b, where the distribution continued to broaden with increasing strain after the initial narrowing. This effect is also visible in the details of the disorientation plot in Fig. 5a (and inset). The retrograde trend in misorientation may result from reorganization and clustering of dislocations into cell boundaries, as discussed later.

The patch of microstructure shown Fig. 6 illustrates examples of slip bands, boundaries with correlated slip transfer, and grain boundary ledge formation. Because large orientation gradients 


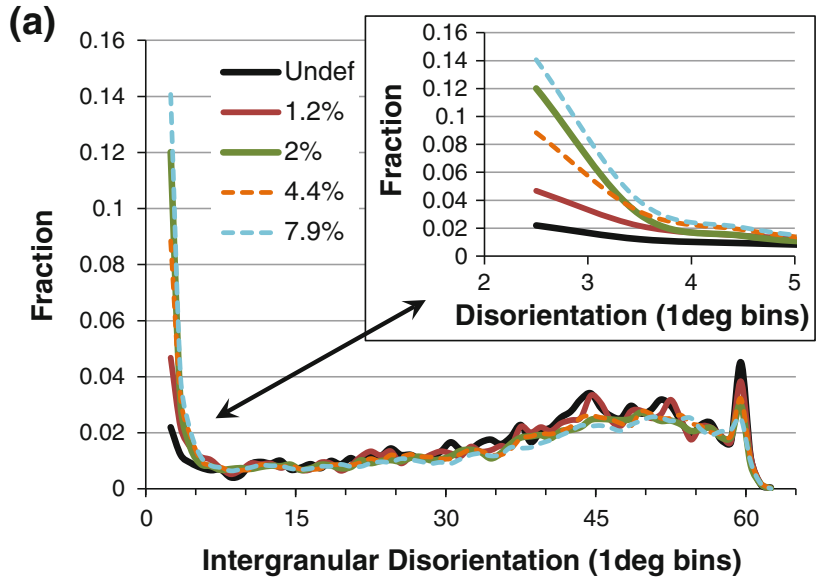

(b)

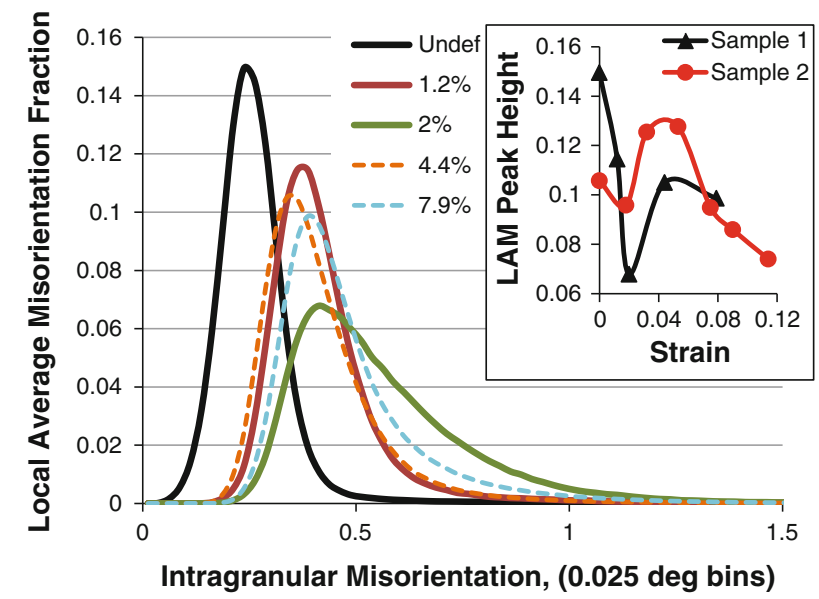

Fig. 5. Intergranular disorientation (a) and intragranular misorientation (b) histograms for several strain values. Low-angle boundaries increase at the lowest disorientation values, and the distribution becomes smoother with increasing strain. The intragranular LAM distribution (based on second nearest-neighbor pixels $700 \mathrm{~nm}$ apart) shows a nonmonotonic progression; see text.

developed in most grains leading to the slip bands often being curved, the assessment of activated slip systems is not straightforward. Active deformation systems were determined by carrying out slip trace analysis at multiple points along curved slip traces based on the locally measured orientations. The eight most highly stressed slip systems based on the global uniaxial stress were identified with different line styles that represent the Schmid factor ordering and were overlaid on Fig. 6. Naturally, the Schmid factor on a particular slip system varies from place to place in the grains because of the orientation variations. A total of 228 identified slip systems from several locations in each of 30 grains in the upper right region of Figs. 3 and 4 are compared to the population of the eight most highly stressed slip systems at each location. Both populations were binned by Schmid factor and plotted in Fig. 7a. The ratios between the two populations are plotted in Fig. 7b, which shows a consistent trend of $\{110\}$ slip traces being slightly more frequently observed than

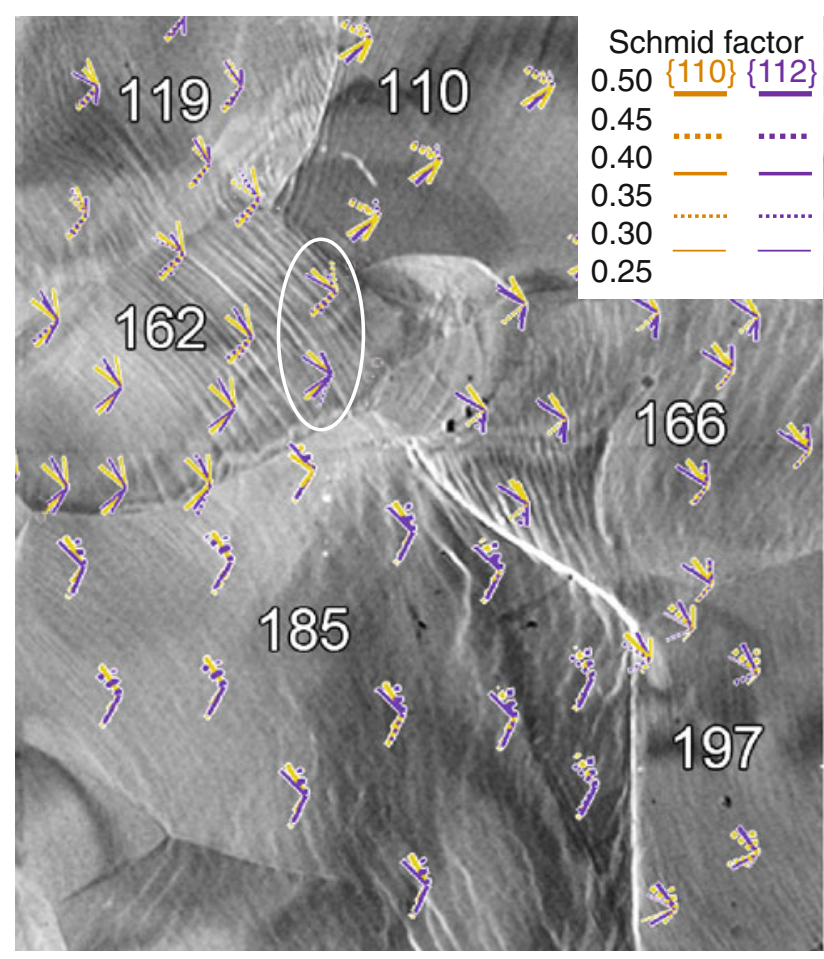

Fig. 6. Secondary electron image with overlaid slip traces based on the local crystal orientation of the eight slip systems with the highest Schmid factors computed using uniaxial stress. The same slip trace in different parts of the grain can have different Schmid factors, e.g., circled pair. Strain is 7.9\%, and location is indicated in Figs. 2 and 4.

$\{112\}$ slip traces. This normalized histogram in Fig. $7 \mathrm{~b}$, which considers the effect of the texture present in the investigated patch, follows an approach developed by Li et al. ${ }^{16}$

To assess the slip transparency of grain boundaries that either display correlated slip transfer or ledge formation, the geometric relationship between likely activated slip systems is examined using the $m^{\prime}$ parameter. Based on average grain orientations from the reconstructed boundary information, values of $m^{\prime}$ were computed for all $24 \times 24=576 \mathrm{slip}$ system combinations across a boundary. Up to the three highest $m^{\prime}$ values were averaged,** provided that their Schmid factors ( $m$, based on uniaxial tension) exceed 0.4. An example of this approach is shown in Fig. 8, where unit cells illustrate two highly stressed slip systems in each grain that result in three high $m^{\prime}$ values. The corresponding table shows how these $m^{\prime}$ values were identified. This computation was done for every grain boundary, resulting in the map in Fig. 9, where colors on the grain boundaries are keyed to the averaged $m^{\prime}$ values (the gray scale identifies the value of the LAM at each pixel). This map allows a comparison between the observed instances of slip transfer and ledge formation. The boundary between grains 119

\footnotetext{
**In some cases there were less than three combinations of slip systems with both $m$ values greater than 0.4 .
} 

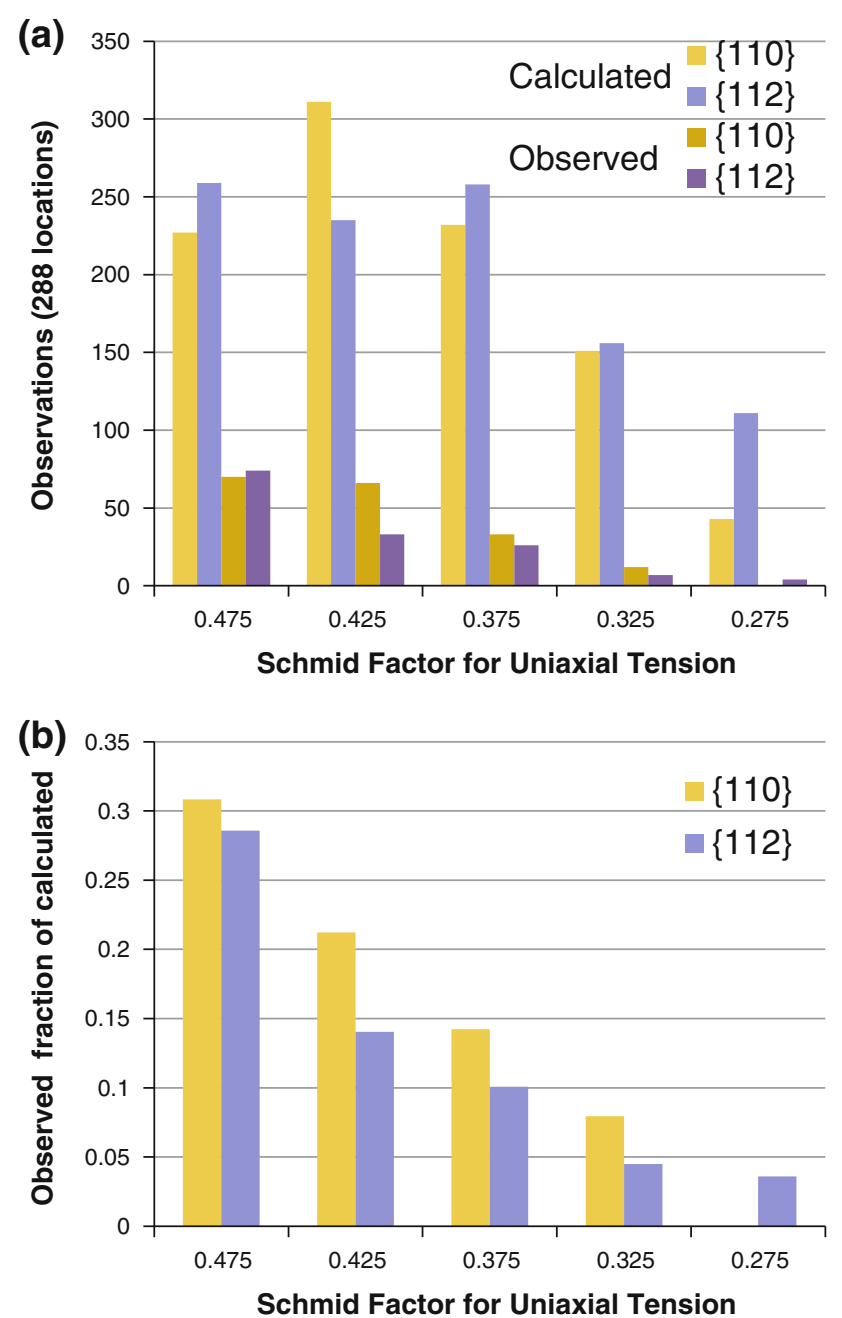

Fig. 7. Slip band analysis of 30 grains in the neighborhood of Fig. 6 , involving 228 specific locations where the local orientation was used to compute Schmid factors and related slip traces. Observed slip bands are plotted along with calculated instances of high Schmid factor slip systems (a). The observed fraction of possible slip behavior is plotted in (b), showing a systematic trend of $\{110\}$ slip being slightly favored over $\{112\}$ slip.

and 162 in Fig. 6 shows correlated slip transfer and a high $m^{\prime}$ value of 0.92 . The boundary between grains 185 and 197 has a lower $m^{\prime}$ value of 0.84 , and it developed a ledge. The correlation between $m^{\prime}$ values and such observations was categorized for 64 boundaries using a scale from 0 to 5 , where 0 indicates either no apparent slip transfer or no ledge and 5 represents obvious slip transfer or an obvious ledge. These results are plotted in Fig. 10a, b. Despite considerable spread, a clear trend indicates that when $\mathrm{m}^{\prime}$ is large, slip transfer is likely and ledge formation is unlikely.

Another way to assess the significance of $m^{\prime}$ is to consider whether dislocation pileup occurs at grain boundaries. The orientation deviation map inset in Fig. $4 \mathrm{~b}$ shows that orientation deviations change monotonically with distance to the grain boundary, implying that orientation gradients are most likely

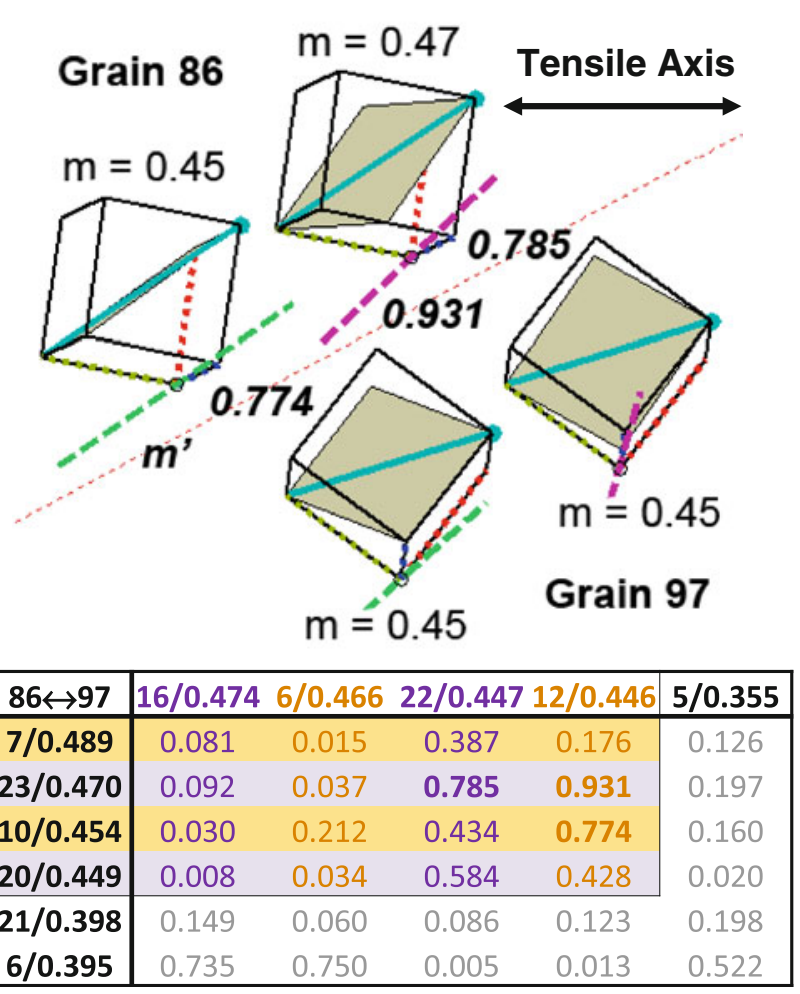

Fig. 8. Visualization of a grain boundary $m^{\prime}$ analysis: Unit cells show slip planes and directions of highly stressed slip systems. The dotted red, yellow, and blue edges on the cube represent the crystal $x, y, z$ directions, and the dashed line represents the plane trace for this slip system. The slip system number/Schmid factor $(m)$ for uniaxial tension is indicated in the top row and left column and sorted by Schmid factor. Slip systems with gold indicate $\{110\}$ slip and purple indicate $\{112\}$ slip. The top three $m^{\prime}$ values greater than 0.6 for slip systems with Schmid factors greater than 0.4 are averaged to provide a measure of the slip transparency of the boundary.

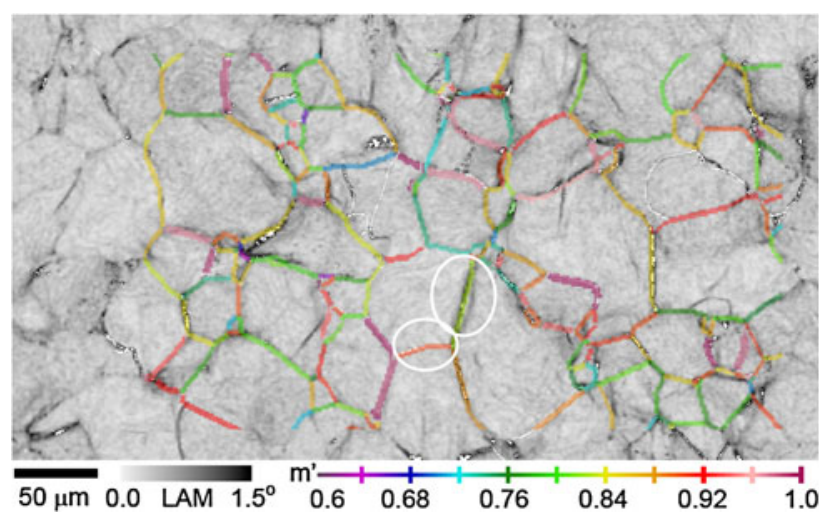

Fig. 9. The values of $m^{\prime}$ on grain boundaries (based on uniaxial tension and average grain orientations) using the method illustrated in Fig. 8 are plotted using the reconstructed grain boundary map from the $7.9 \%$ strain data. The information plotted within the grains is the LAM on a gray scale (compare with Fig. $4 \mathrm{~b}$ ). The correlation between low $m^{\prime}$ and higher LAM values at grain boundaries is plotted in Fig. 10c.

due to accumulated dislocations of the same type. Piled up dislocations generally cause orientation gradients near grain boundaries, so the LAM values 
(a)

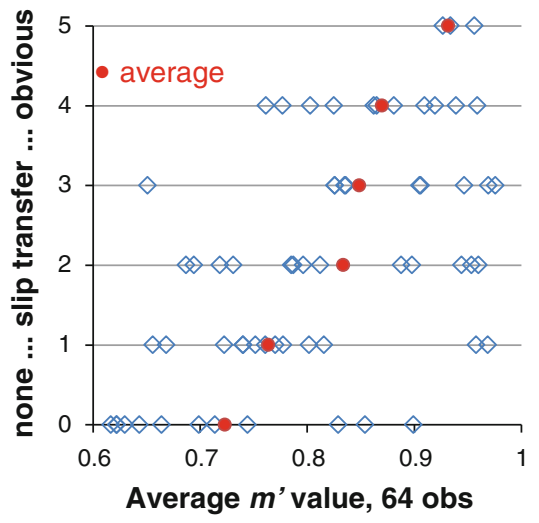

(b)

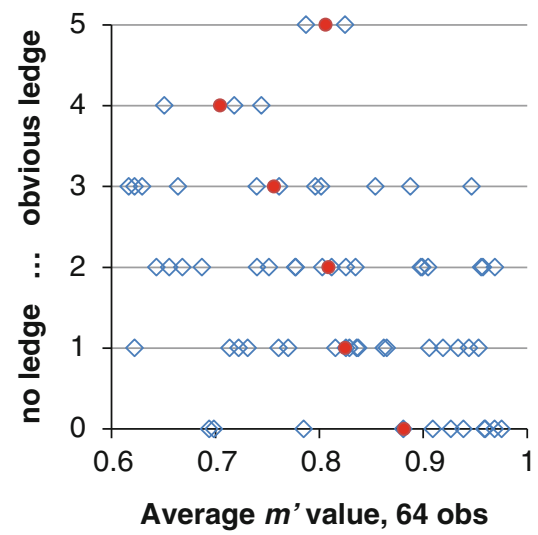

(c)

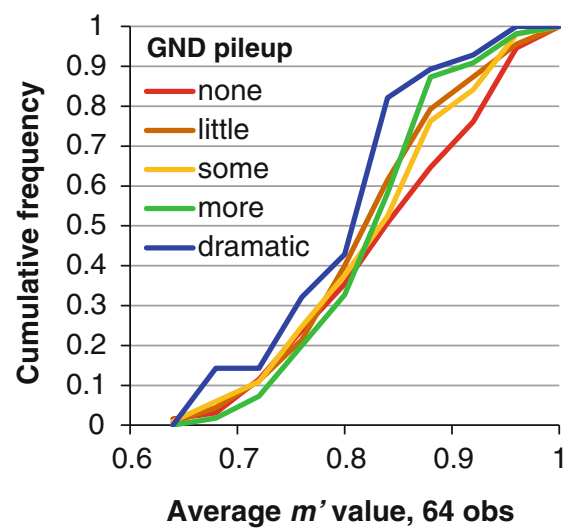

Fig. 10. Correlations of obvious slip transfer (a), ledge formation (b), and GND accumulation at grain boundaries (c), with respect to $m^{\prime}$ values. Plots are semiquantitative, based on $(a, b)$ visual assessment of SEM surface and (c) LAM maps.

can be used to determine whether slip transfer was frustrated. Figure 9 illustrates a LAM map for the grain interiors expressed as a gray scale overlaid with colored $m^{\prime}$ values on boundaries. There are several examples where LAM values are high adjacent to boundaries and others where the LAM is low. When the same 64 boundaries considered for the above $m^{\prime}$ analysis are evaluated, there is evidence that high $m^{\prime}$ values are correlated with conditions where no accumulation of geometrically necessary dislcoations (GNDs) are observed, but dramatic accumulation of GNDs at a boundary are correlated with low $m^{\prime}$ values, as shown in Fig. 10c.

\section{DISCUSSION}

These results provide information that allows physical rationale that can account for the varying deformation behavior of grain boundaries. While there is significant scatter in the data, some correlations suggest that physically based criteria for grain boundary character evolution that precede damage nucleation can be described and implemented into physically based models.

The deformation of Ta polycrystals develops with early strain in a complex manner as indicated by the nonmonotonic evolution of the LAM data. The fact that the peak did not decrease and monotonically move to larger angle values indicates that dislocation substructure evolved heterogeneously at very early stages of deformation. This indicates that some form of dislocation rearrangement took place at low strains, such that some regions that once had lattice curvature became less populated with dislocations. This is consistent with the increase in lowangle boundaries, where dislocations accumulated into low-angle boundaries or walls in this range of strain. Further increases in strain led to developing larger gradients in the dislocation wall regions and, hence, spreading the distribution to higher angles. It is likely that these processes set up the local structures that cause development of large-scale orientation gradients that become more obvious with larger strains.

Another important component of understanding substructure evolution is to identify the relative activity of the $\{110\}$ and $\{112\}$ slip system families. The role of screw dislocations on limiting the progress of slip in bcc metals is well known. Given that screw dislocations readily cross slip, the slip lines observed in bcc metals are often wavy, leading to difficulty in interpreting the very obvious slip bands that are apparent on polished surfaces after only small amounts of strain. This ease of cross-slip, combined with the core structure of $\langle 111\rangle$ screw dislocations in bcc metals, can result in the dislocations weaving between $\{110\}$ and $\{112\}$ planes; slip traces can lie anywhere in between the slip traces for the two conjugate $\{112\}$ planes (wavy slip). However, in this study, there is clear evidence that many slip bands are quite distinct, such as the bands in grain 162 in Fig. 6, which change direction gradually in ways that are consistent with orientation gradients in the grain. There is also evidence for wavy slip such as in the lower parts of grain 185 and the right side of grain 166 .

Although this analysis shows significant correlations between different types of grain boundary slip interactions and the geometric activity and alignment of slip systems, there is considerable scatter in the analysis. This scatter can arise from several sources. First, it is clear from the orientation gradients that develop even at very small strains that strain in most of the grains is nonuniform and highly influenced by deformation in neighboring crystals. This implies that the local stress tensor varies from the global stress tensor in a complex way. This fact creates significant uncertainty in interpreting experimental results with the simple assumption of uniaxial stress. This is consistent with the fact that, in many cases, the slip system with the highest (global) Schmid factor was not 
observed. Second, it is possible that slip systems with Burgers vectors close to parallel with the sample surface did not develop recognizable slip bands. Although such slip systems are hard to observe, they were not excluded from the Schmid factor and $m^{\prime}$ analysis. Hence, it is possible that slip transfer occurred in a nonobvious way. Similarly, spatially uniform slip, as opposed to slip concentrated in bands, may not be visible even if very active. Third, the Schmid factor and $m^{\prime}$ analysis presented in this study is based on the average grain orientation not the actual orientation present on either side of the boundary. Because of the orientation gradients within grains, the values of $m^{\prime}$ and $m$ consequently are not precise for the actual conditions at the grain boundary. Thus, it is not surprising that there is considerable spread in Fig. 10 due to the necessary semiquantitative approximations, so statistical sampling is required for obtaining a trend from conditions that have many other co-factors.

All of these considerations also imply that improved interpretations of experimental work could be effectively informed by correlating simulations of microstructure using crystal plasticity models in connection with finite element or spectral solvers. ${ }^{10,17}$ However, developing the representations of the actual microstructure requires its nondestructive three-dimensional (3-D) characterization prior to and after deformation (which is not trivial). Nevertheless, the current analysis provides an indication of mechanistic phenomena that take place at the mesoscale despite the lack of credible stress tensor information. There are clear indicators that slip transfer occurs in some boundaries but not others, in ways that are consistent with the geometrical relationship of slip systems in each grain.

\section{CONCLUSIONS}

An analysis of EBSD data taken at multiple strains during three-point bending of tantalum polycrystals led to the identification of mechanisms related to heterogeneous deformation, which is a precursor to damage nucleation.

1. Heterogeneous strain commenced at strains as low as $1 \%$; with increasing strain, orientation gradients that exceeded $10^{\circ}$ developed within most grains.

2. Up to strains of about $2 \%$, the local average misorientation distribution became broader, indicating increasing amounts of lattice curvature through dislocation accumulation. With further straining, the peak became sharper as subgrain formation led to clustering of dislocations. With further straining past about $5 \%$, the distribution broadened thereafter in a monotonic way.
3. A careful slip trace analysis of slip bands using local orientation data indicated that both $\{110\}$ and $\{112\}$ slip planes were active during deformation.

4. Correlations between the Schmid factor, the slip transfer parameter $m^{\prime}$, and observations of correlated slip bands matching up on both sides of the boundary were identified. In cases where the slip system geometry was not favorable for slip transfer, ledges between grains were more likely to develop. Boundaries not geometrically favorable to slip transfer were more likely to develop dislocation pileups on one side and/or the other.

5. Considerable uncertainty about the local stress tensor leads to scatter in measurements, so accurate 3 -D crystal plasticity simulations that identify the actual partitioning of stress among neighboring grains could enhance the interpretation and analysis of heterogeneous deformation investigations.

\section{ACKNOWLEDGEMENTS}

Sandia National Laboratories is a multiprogram laboratory managed and operated by Sandia Corporation, a wholly owned subsidiary of Lockheed Martin Corporation, for the U.S. Department of Energy's National Nuclear Security Administration under contract DE-AC04-94AL85000.

\section{REFERENCES}

1. T.R. Bieler, P. Eisenlohr, F. Roters, D. Kumar, D.E. Mason, M.A. Crimp, and D. Raabe, Int. J. Plast. 25, 1655 (2009).

2. M.D. Sangid, T. Ezaz, H. Sehitoglu, and I.M. Robertson, Acta Mater. 59, 283 (2011).

3. X. Wael, Z. Abuzaid, M.D. Sangid, J.D. Carroll, H. Sehitoglu, and J. Lambros, J. Mech. Phys. Solids 60, 1201 (2012).

4. J. Kacher and I.M. Robertson, Acta Mater. 60, 6657 (2012).

5. T.B. Britton, D. Randman, and A.J. Wilkinson, J. Mater. Res. 24, 607 (2009).

6. C. Brandl, E. Bitzek, P.M. Derlet, and H. Van Swygenhoven, Appl. Phys. Lett. 91, 111914 (2007).

7. Z. Shen, R.H. Wagoner, and W.A.T. Clark, Acta Metall. 36, 3231 (1988).

8. J. Luster and M.A. Morris, Metall. Mater. Trans. A 26, 1745 (1995).

9. L. Wang, Y. Yang, P. Eisenlohr, T.R. Bieler, M.A. Crimp, and D.E. Mason, Metall. Mater. Trans. A 41A, 421 (2010).

10. L. Wang, R. Barabash, Y. Yang, T. Bieler, M. Crimp, P. Eisenlohr, W. Liu, and G. Ice, Metall. Mater. Trans. A 42A, 626 (2011).

11. C.R. Weinberger, B.L. Boyce, and C.C. Battaile, Int. Mater. Rev. 58, 296 (2013).

12. A. Seeger and U. Holzwarth, Philos. Mag. 86, 3861 (2006).

13. D. Raabe, G. Schlenkert, H. Weisshaupt, and K. Lücke, Mater. Sci. Technol. 10, 299 (1994).

14. J.D. Carroll, B.G. Clark, T.E. Buchheit, B.L. Boyce, and C.R. Weinberger, Mater. Sci. Eng. A 581, 108 (2013).

15. B.L. Boyce, B.G. Clark, P. Lu, J.D. Carroll, and C.R. Weinberg, Metall. Mater. Trans. A 44A, 4567 (2013).

16. H. Li, D.E. Mason, T.R. Bieler, C.J. Boehlert, and M.A. Crimp, Acta Mater. 61, 7555 (2013).

17. P. Eisenlohr, M. Diehl, R.A. Lebensohn, and F. Roters, Int. J. Plast. 46, 37 (2013). 